\title{
IFU spectroscopy of the LINER NGC 7213
}

\author{
Alexandre S. Oliveira ${ }^{1}$ and J. E. Steiner ${ }^{1}$ \\ ${ }^{1}$ IAG/USP, São Paulo, Brazil email: alex and steiner@astro.iag.usp.br
}

\begin{abstract}
We made observations of the LINER NGC 7213 in order to perform critical procedures in observing with the Eucalyptus IFU spectrograph. This object has emission lines with stellar profiles surrounded by weak extended nebular emission. We obtained narrow bands images and space resolved spectra, which suggest the existence of a bar of stellar origin.
\end{abstract}

\section{Introduction - The Eucalyptus spectrograph}

With the new generation of modern telescopes that have as an important goal to offer superb image quality, it is important to develop instruments that can deliver spectroscopic data with optimal space resolution. One of the roads towards achieving this is by IFU spectroscopy. The goal of this work is to perform critical procedures in observing and reducing the data obtained with the Eucalyptus IFU spectrograph. For this purpose we observed NGC 7213 - a prototype LINER galaxy with broad $\mathrm{H} \alpha$ emission. The Eucalyptus (de Oliveira et al. 2003) is a prototype of the SIFS (SOAR Integral Field Spectrograph) and is currently operational at the Observatório Pico dos Dias (OPD), Brazil. It is composed of a matrix of $16 \times 32$ lenslets and covers a field of $15 \times 30^{\prime \prime}$.

\section{Narrow emission line images and space resolved spectra}

The LINER NGC 7213 has emission lines with stellar profiles surrounded by weak extended nebular emission, and the images in narrow bands are quite useful in interpreting it. The nuclear AGN is superposed onto a stellar population (Fig. 1). By subtracting the stellar continuum from the narrow emission, we obtain the $\mathrm{H} \alpha$ image with a narrow (4A) band-pass. Similar images can be produced at other emission lines such as [N II] and $[\mathrm{O} \mathrm{I}]$, all illustrated in Fig. 1. Each image has a profile that can be characterized by FWHM as shown in Table 1, which shows that the LINER nucleus has a circular image with (instrumental) resolution of about $\mathrm{FWHM}=2.6^{\prime \prime}$. In white light, however, the right ascension dimension is $5.1^{\prime \prime}$. This may suggest the existence of a bar of stellar origin oriented along the right ascension. Another way that IFU spectroscopy can reveal useful is in producing space resolved spectra (Fig. 2). They show that NGC 7213 has little if any resolved emission. Storchi-Bergmann et al. (1996) found significant $\mathrm{H} \alpha$ emission at a radius of about $25^{\prime \prime}$ from the nucleus and week emission near the nucleus. Their space and spectral resolution are somewhat poorer then ours and certainly have higher signal-to-noise, which may explain this discrepancy.

\section{References}

de Oliveira, A. C., et al. 2003, SPIE.4841.1417

Storchi-Bergmann, T., Rodriguez-Ardila, A., Schmitt, H. R., Wilson, A. S., \& Baldwin, J. A. 1996, ApJ, 472, 83 


$\begin{array}{lcc}\text { source } & \text { right ascension } & \text { declination } \\ \text { White light } & 5.1(0.2) & 2.8(0.2) \\ \mathrm{H} \alpha \text { narrow } & 2.7(0.2) & 2.4(0.2) \\ \mathrm{H} \alpha \text { broad } & 2.4(0.3) & 2.2(0.3) \\ {[N I I]} & 2.9(0.2) & 2.7(0.2) \\ {[O I]} & 2.3(0.3) & 2.3(0.3)\end{array}$

Table 1. FWHM at various narrow bands

Fig. 1

Narrow band images of NGC 7213

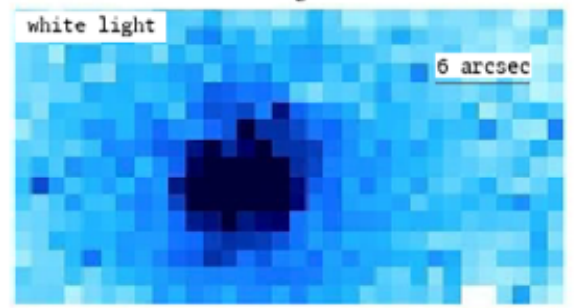

Fig. 2
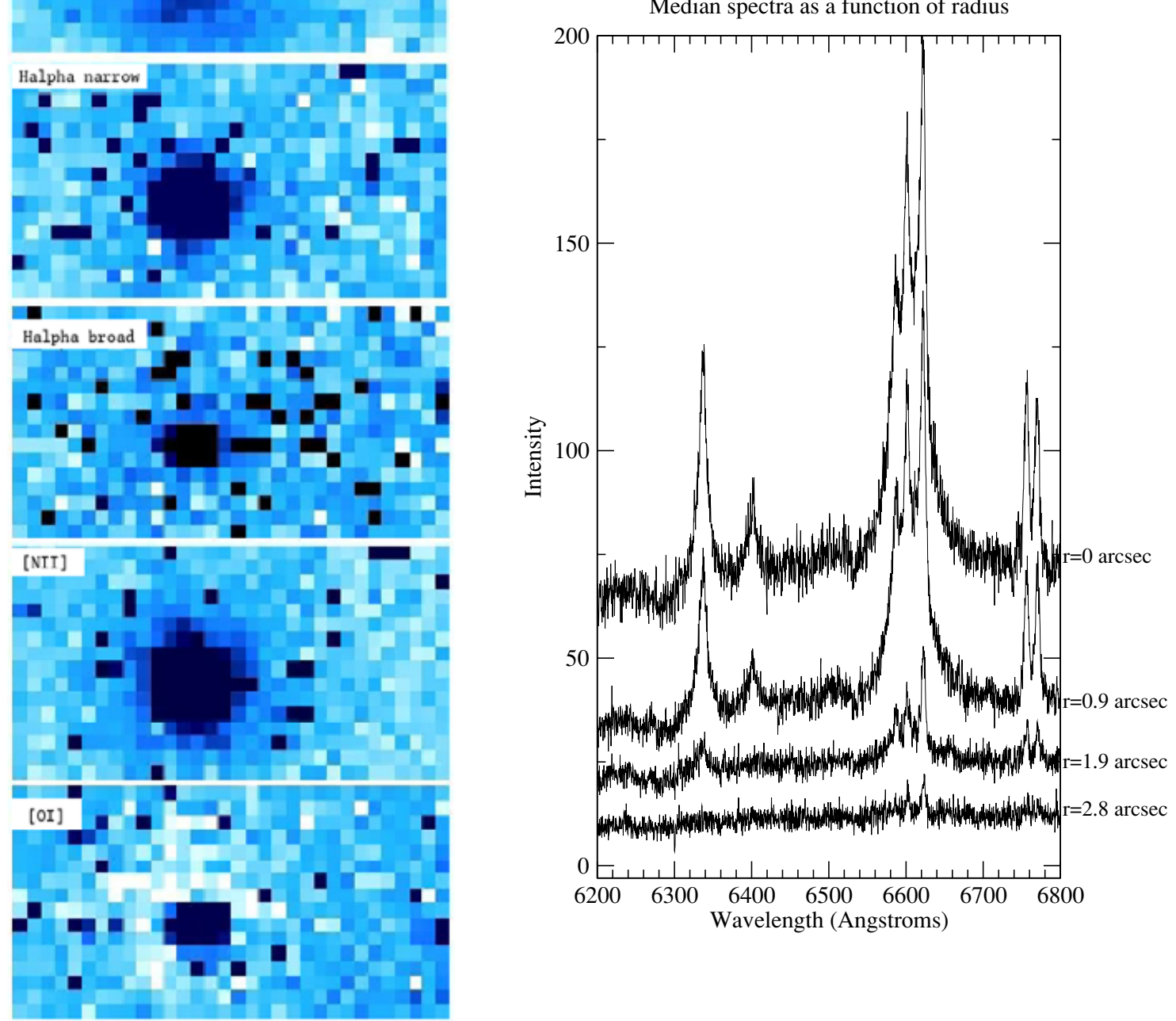